\title{
Identification of Self-Organized Critical State on Twitter Based on the Retweets' Time Series Analysis
}

\author{
Andrey Dmitriev (iD) and Victor Dmitriev iD \\ Department of Business Informatics, National Research University Higher School of Economics, Moscow, Russia \\ Correspondence should be addressed to Andrey Dmitriev; a.dmitriev@hse.ru
}

Received 15 October 2020; Revised 2 December 2020; Accepted 11 January 2021; Published 20 January 2021

Academic Editor: Tingqiang Chen

Copyright (C) 2021 Andrey Dmitriev and Victor Dmitriev. This is an open access article distributed under the Creative Commons Attribution License, which permits unrestricted use, distribution, and reproduction in any medium, provided the original work is properly cited.

\begin{abstract}
There is a number of studies, in which it is established that the observed flows of microposts generated by microblogging social networks (e.g., Twitter) are characterized by avalanche-like behavior. Time series of microposts depicting such streams are the time series with a power-law distribution, with $1 / f$ noise and long memory. Despite this, there are no studies devoted to the detection and analysis of self-organized critical state, subcritical phase, and supercritical phase. The presented paper is devoted to the detection and investigation of such critical states and phases. An algorithm is proposed that allowed to detect of critical phases and critical conditions on Twitter, based on the analysis of retweets time series corresponding to the three debates of the 2016 United States Presidential Election, as the most popular debate in the history of America, collecting 84 million live views.
\end{abstract}

\section{Introduction}

One of the most relevant and promising areas of modern science is the usage of models and methods of complex systems' physics in the analysis of economic, historical, demographic, and political phenomena.

The most significant results of recent years in the study of network structures have been obtained by physicists. It turned out that the methods of theoretical physics are the most successful for describing a wide class of critical phenomena in networks due to their self-organization (e.g., see the review [1]). Such critical phenomena in networks can occur either as a result of fine-tuning of the control parameter until it reaches a critical value, or spontaneously, without requiring fine-tuning of the control parameter.

Systems' self-organization into the critical state that requires fine-tuning of the control parameter includes selforganization into the critical state during phase transitions. Such self-organization, which is characterized by spatial and scale invariance, occurs when the control parameter reaches its critical value. There are several studies on phase transitions on social networks (e.g., see the papers [2-8]). The application of phase transition of topics for event detection on Twitter was studied by Barros and coauthors [9]. Another example of this type of critical phenomena is the percolation phase transition, which is realized as a result of fine-tuning the fraction of nodes until it reaches a critical value, at which a scale-invariant percolation cluster arises. Studies of the self-organization of the social networks into the critical state as a result of percolation phase transitions are presented in [10-15]. For example, the reaction of Twitter users to the scientific knowledge percolation process was studied by B. Barros and coauthors [16].

Parameter's fine-tuning to its critical value is possible only under laboratory conditions, but not under natural conditions. For example, such tuning is not possible in complex dynamic systems such as social networks. However, Bak was the first to show that it is possible that many complex dynamical systems self-organize in a critical point regardless of their initial states in [17]. In a certain sense, such systems present self-organized criticality (SOC). Being in a chaotic subcritical phase (SubC phase), such dynamic systems naturally evolve to the SOC state with the possibility of further transition to an ordered supercritical phase (SupC phase). In a SOC state, a small event (impact) triggers a chain reaction that can affect any number of system elements. Avalanches of all sizes are observed in the SupC phase and near the SOC state; like clusters of all sizes arise when a 
percolation cluster occurs. Such avalanches do not depend on the magnitude of external influences or fluctuations. Even a very small external impact can lead to an avalanche of enormous size.

The nonlinear dynamics of the SOC occurrence on Twitter can be qualitatively described in the same way as the occurrence of the SOC in the growing sand pile. The sand pile model is often used as a visual illustration of the selforganization of open dissipative systems into the critical state (e.g., see the papers $[18,19])$. The description of the SOC occurrence in the sand pile is the simplest when we need to describe the features of the time variation of the macroscopic order parameter $\left(\eta_{t}\right)$ and the control parameter $\left(S_{t}\right)$. To describe Twitter's self-organization into the critical state, we will denote the number of retweets as $\eta_{t}$ and the number of primary sources (users) of the retweets' distribution on the network as $S_{t}$ (see Section 4). In the sand pile model, $\eta_{t}$ is the integral sand current from the surface of the pile and $S_{t}$ is the angle of the sand pile. According to the model, starting from time $t=0$, one sand grain falls to the top of the pile every time interval $\Delta t \sim 0$. In some places of pile, where the local slope is greater than the stability threshold, shedding occurs. This causes that sand grains slide down the slope to adjacent areas of the pile surface. In the time interval $\left(0, t_{c}\right)$, corresponding to the chaotic SubC phase, $\eta_{t<t_{c}}=0$. The absence of current, observed when $S_{t}<S_{t}$, is the result of the interaction of a set of chaotically directed mutually balanced microcurrents. At the time $t \sim t_{c}$, corresponding to the unstable SOC state, when $S_{t}$ reaches its critical value $S_{t_{c}}$, a dedicated current direction appears $\left(\eta_{t \geq t_{c}}>0\right)$. If $\eta_{t \sim t_{c}}=0^{+}$, then when one sand grain is added in one time interval $\Delta t \sim 0$, the pile goes into the SOC state, in which $S_{t}$ is tuned to $S_{t}$. This distinguishes the SOC phenomenon from a conventional continuous phase transition. Finally, being in the ordered SupC phase at $t>t_{c}$, the sand avalanches of any size fall from the surface of the pile.

Recently, there has been strong evidence of the existence of the SOC state on the social networks that is presented in the number of fundamental studies (e.g., see the papers [20-25]). The following papers are the most relevant to our research. Tadic and coauthors [26] proved the presence of the SOC in the Internet communities and established mechanisms of the SOC in social processes of knowledge creation. Shimada and Koyama [27] put forward and substantiated the assumption that the appearance of $1 / f$ noise in electoral statistics may indicate the readiness of certain groups of voters for a radical political transformation. Antoniades and Dovrolis [28] studied coevolutionary dynamics on Twitter. Casero-Ripolles [29] identified "the key points of the transformations that social media have generated in the field of political information." In contrast, Stromer-Galley states in her book [30] that "the need for communication control and management has made campaigns slow and loathe to experiment with truly interactive Internet communication technologies." Barabash and Zhukov [31] proposed an approach to identify political mobilization on the social networks based on the time series analysis.
The emergence of the SOC on the social networks is evidenced by the avalanche-like dynamics of microposts observed in them [32-39].

Despite the numerous studies of social networks evolution, the study of the avalanche-like behavior in them is carried out for the entire empirical sample without dividing it into intervals (critical phases). This allows us to establish only the existence of the SOC in the evolution of the social network but leaves open the important question of the time intervals existence for its evolution: the chaotic SubC phase and the ordered SupC phase, as well as the moment of the formation of the SOC state. The detection of such states and phases of Twitter based on the analysis of retweet activity on the social network is the purpose of our study.

The choice of Twitter as the research object is not accidental. Over the past years, Twitter has attracted more and more attention not only from sociologists, but also from political scientists, virologists, and geophysicists. For example, real-time tweet flow analysis for predicting trends (e.g., see the papers $[40,41])$, public sentiment (e.g., see the papers $[42,43])$, election results (e.g., see the papers [44-48]), prompt detection of important events (e.g., see the paper [49]), sources of viral infections (e.g., see the paper [50]), and earthquakes and typhoons (e.g., see the paper [51]).

\section{Data Set and Methods}

Most critical phenomena models in complex networks are based on the analysis of changes in their structure as a result of the external influences (e.g., see the review [52]). In most cases, models of equilibrium and nonequilibrium statistical physics are used to describe critical phenomena on the social networks. The study of critical phenomena on the social networks at the structural level is the most informative for describing microscopic interactions between network users. In the case where such a description is necessary, the study of the social network at this level is most appropriate. Despite this, verification of such models requires access to the structural data of the social network, which are not always publicly available and in some cases are not available at all. Despite this, verification of such models requires access to the structural data of the social network, which are not always publicly available and in some cases are not available at all.

We recently proposed the method for studying critical phenomena at the level of external manifestations of the social network. It is based on statistical and spectral analysis of the time series (e.g., micropost time series) generated by the social network (see the paper [53]). This method does not require empirical information about structural changes on the social network, but despite its simplicity, it allows to determine the time intervals of critical phases and the time at which the network reaches the SOC state. This is quite enough to achieve the goal of our study.

A brief description of the method, as well as the specifics of determining the order parameter and the control parameter, is presented in Section 2.2. The way to extract the data needed to receive the retweet time series is presented in Section 2.1. Finally, an algorithm for identifying critical phases and the SOC state is presented in Section 2.3. 
2.1. Data Set for Retweet Time Series. The activity of communities, including the electorate, demonstrates several different time series on the social networks. For example, such activity can be demonstrated by the dynamics of the number of likes, the dynamics of visits and views, and some other dynamics. In our opinion, the most suitable time series for identifying the time intervals of critical phases and the time of self-organization in a critical state is the retweet time series. Such time series represents the dynamics of messages that network users perceived and distributed among other users, their subscribers. Retweet is a fundamental act of reflection on the social network. The appearance of the retweet on the network means that the information is reflected by the user and reproduced among other users. That is why retweets are a way of spreading information on microblogging social networks. They give opportunity to the social networks to influence the opinions, ratings, and behavior of their users. Finally, we can judge the presence/absence of electoral mobilization or electoral self-organization by the type of retweet activity and without referring to the empirical structural data of the social network. Thus, Nishi and coauthors state in their paper [54] that "in a network of posts, an initial post located at the root of the network may induce a cascade of responses of different magnitudes and spatiotemporal patterns."

We hydrated the tweet IDs (2016 United States Presidential Election Tweet IDs [55]), which were obtained from the Harvard Dataverse using the Social Feed Manager, to form the Twitter time series. Before getting the Twitter time series, we filtered out the "technical" objects that link to blocked users at the time of the research. Considering that each object contains information about the corresponding number of retweets, we additionally selected only "original" user tweets. To get the retweet time series, the numeric values of the "retweet_count" field were summed up for all "original" tweets that have the same timestamp. The values for the initial micropost time series were obtained by calculating the sum of the "original" tweets with the same timestamp.

As a result, we got three equidistant (a step is 1 second) time series of retweets $\left(x_{i}, i=1, \ldots, T\right)$ of different lengths, each of which is relevant to some topic (1st debate, 2nd debate, and 3rd debate). We obtained two retweet time series classes corresponding to three debates: the time series showing the change in the number of retweets and the time series showing the change in the number of initial microposts for each chain of retweets.

2.2. Identifiers of Critical Phases. We have previously shown in [53] that the Langevin equation for the order parameter with nonlinear drift and diffusion parts is a good approximation for describing the stochastic dynamics of microposts on Twitter. This equation is based on the fundamental principles of the social network functioning. The study of the equation solutions made it possible to introduce interval identifiers for the SubC phase and the SupC phase and substantiate their applicability for detecting the presence of the social network in one of the phases based on the analysis results of the empirical time series. Interval identifiers or interval spectrum of criticality indicators for time series is a set of scaling exponents $\{\alpha, \beta, \gamma\}$ of power laws for probability density function (PDF), power spectral density (PSD), and autocorrelation function (ACF). Justification of the applicability of the spectrum $\{\alpha, \beta, \gamma\}$ as an identifier of critical phases, as well as the number of spectrum approbations on the real data, is presented in the paper [53]. Therefore, we will restrict ourselves to a brief description of this method below.

The most suitable macroscopic value, according to the analysis of which the critical phases can be identified, is the order parameter $\eta$. For the chaotic SubC phase $\eta=0, \eta \neq 0$ for the ordered SupC phase. Calculation of $\eta$ as the average over the statistical assembly $\eta=\sum \eta(r, t) / \mathcal{N}$ for a large number of $\mathcal{N}$ retweets requires specifying dynamic variables $\eta(r, t)$. For example, $\eta(r, t)$ can characterize the direction of the retweet in space. If we limit ourselves to the study of the static critical phenomena that occur in equilibrium systems, it is permissible to calculate the order parameter using the functions $\eta(r)$. The study of dynamic critical phenomena is a more complex problem, since it requires the computation of the functions $\eta(r, t)$, and the computation of temporal fluctuations of the order parameter or some other macroscopic quantities. Considering that the purpose of our study is to detect time intervals for critical phases and the time of Twitter's self-organization to the critical state, it is quite sufficient to carry out statistical and spectral analysis of multiple time intervals for the retweet time series $\left(x_{t}\right)$. We used the number of initial microposts as the control parameter $\left(S_{t}\right)$. This parameter determines the number of primary sources (for example, influencers) that initiate the distribution of retweets chain on the social network. Such a chain corresponds to a hierarchically organized scheme of information distribution on the network: an influential user $\longrightarrow$ his subscribers $\longrightarrow$ subscribers of their subscribers, etc.

The segment of the social network is in the SupC phase for some time $\Delta t_{\text {SupC }}$; if power laws are fulfilled for $\Delta t_{\text {SupC }}$, retweet time series with indicators belonging to the intervals from Table 1:

$$
y \propto x^{-\delta}
$$

where $y \equiv p, \delta \equiv \alpha$ for PDF, $y \equiv S, \delta \equiv \beta$ for PSD, and $y \equiv \rho, \delta \equiv \gamma$ for ACF.

The ordered SupC phase in network is characterized by power law for PDF, PSD with $1 / f$ noise, and long temporal correlations. In contrast, the chaotic SubC phase is characterized by a compact probability distribution (e.g., exponential distribution), PSD with white noise $(\beta \cong 0)$, and short temporal correlations (e.g., exponential law for ACF).

It is important to note that a necessary condition for the social network being in the SupC phase is belonging of all three criticality indicators $\{\alpha, \beta, \gamma\}$ to corresponding intervals. Most researchers use only the existence of PSD in the form of $1 / f$ noise as an indicator of SOC. This approach is valid for some random processes and model time series, for which a relationship between all three indicators of criticality 
TABLE 1: Interval features of critical phases. The symbol “_” symbol means a power law failure.

\begin{tabular}{l|cc}
\hline Exponent $\mid$ phase & Subcritical & Supercritical \\
\hline$\alpha$ & - & $(1,3)$ \\
$\beta$ & $\cong 0$ & $(0.5,1.5)$ \\
$\gamma$ & - & $(0,1)$ \\
\hline
\end{tabular}

has been established (e.g., see $[56,57])$. In this case, we can limit by assessing one of the criticality indicators. But, for real time series, such reliable connections have not been established.

The use of the power laws (1) to identify the SupC phase makes it possible to limit ourselves to testing the statistical hypothesis of the significance of a simple linear regression (linearized equation (1)). We used the ordinary least squares method for estimating $\delta$. At the same time, we used $p$ value as an indicator of the minimum level of significance.

Calculation of the spectrum of criticality indicators $\{\alpha, \beta, \gamma\}$ is the main stage for the algorithm for identifying critical phases, the steps of which will be discussed in Section 2.3.

\subsection{Algorithm for Identification of Critical State and Phases.} We introduce the following notation:

(i) $x_{t}, t=0,1,2, \ldots, T$ is equidistant discrete time series of retweets

(ii) $t_{\text {SubC }}^{0}$ is the SubC phase formation start time

(iii) $t_{c}$ is the moment of time corresponding to the SOC state and, accordingly, the start time of the formation of the SupC phase $\left(t_{c} \equiv t_{\text {SupC }}^{0}\right)$

(iv) $t_{\text {SupC }}^{f}$ is the SupC phase completion time

To detect time intervals, which are characterized for the SubC and the SupC phases, as well as a point of time that is characterized for the SOC state, we proposed the following algorithm for analyzing time series of retweets:

(i) Step 1. Calculate the spectrum $\{\alpha, \beta, \gamma\}$ for the time series at $t \in[0, T]$.

The calculation of the spectrum $\{\alpha, \beta, \gamma\}$ at this step allows us to determine the existence or absence of the SupC phase. The existence of such a phase is the necessary but not sufficient condition for the existence of the SOC state at $t \in[0, T]$. If for the entire length of the time series $(t \in[0, T])$ the spectrum of criticality indicators $\{\alpha, \beta, \gamma\} \notin\{(1,3),(0.5,1.5),(0,1)\}$, then the system, generating this time series, is in the SubC phase. Therefore, there is no need to perform the following steps of the algorithm for the available empirical data. If for the time series the spectrum $\{\alpha, \beta, \gamma\} \in\{(1,3),(0.5,1.5),(0,1)\}$ at a certain level of significance, then we need to go to the next step.

(ii) Step 2. We calculate the set of spectra $\{\alpha, \beta, \gamma\}_{k}, k=T, T-1, \ldots, T-m$, and $p_{k}$ levels for each of the intervals $[0, k]$ by reducing the length of the interval $[0, T]$ in the direction $T \longrightarrow 0$ for the fixed $t=0$. The time series interval is the smallest interval for which the statistically significant dependence is in the log-log scale, at least.

If the SOC state is formed in the system, then the set of spectra $\{\alpha, \beta, \gamma\}_{k} \in\{(1,3),(0.5,1.5),(0,1)\}$ with acceptable $p_{k}$ levels up to a certain point in time $t^{\prime}$ $\left(m<t^{\prime}<T\right)$. Then, with decreasing time $t\left(t<t^{\prime}\right)$, the spectra $\{\alpha, \beta, \gamma\}_{k} \notin\{(1,3),(0.5,1.5),(0,1)\}$. In this case, the time moment $t /$ is a robust lower bound $t_{c}$.

(iii) Step 3. Similarly to the calculation for step 2, we calculate the spectra $\{\alpha, \beta, \gamma\}_{k}, k=T-1, T-2, \ldots, T-l$, and $p_{k}$ levels for each of the intervals $[0, l]$. Unlike the calculations in step 2, all calculations are performed for a fixed $t=T$ in the direction $0 \longrightarrow T$.

If the SOC state is formed in the system, then the set of spectra $\{\alpha, \beta, \gamma\}_{k} \in\{(1,3),(0.5,1.5),(0,1)\}$ with acceptable $p_{k}$ levels up to a certain point in time $t^{\prime \prime}$ $\left(t^{\prime \prime}<T\right)$. Then, with decreasing time $t\left(t>t^{\prime \prime}\right)$, the spectra $\{\alpha, \beta, \gamma\}_{k} \in\{(1,3),(0.5,1.5),(0,1)\}$, but with lower $p_{k}$ levels.

(iv) Step 4. We calculate the critical time as $t_{c}=\left(t^{\prime}+t^{\prime \prime}\right) / 2$, and $\Delta t_{\text {SubC }}, \Delta t_{\text {SupC }}$.

By evaluating $t_{c}$, we can estimate the time intervals corresponding to the SubC phase $\left(\Delta t_{\text {SubC }}=t_{c}-0\right)$ and the SupC phase $\left(\Delta t_{\text {SupC }}=T-t_{c}\right)$.

It is important to note that the applicability of the algorithm is limited to the case when a social network is characterized by the following ordered sequence of phases and state in the process of its evolution: SubC phase $\longrightarrow$ SOC state $\longrightarrow$ SupC phase.

\section{Results}

We have obtained the following retweet time series and initial micropost time series:

(i) From 17:00 on September 26, 2016, to 15:00 on September 27, 2016, with 1-second increment (see Figures 1 and 2)

(ii) From $01: 00$ on October 7, 2016, to $17: 00$ on October 10, 2016, with 1-second increment (see Figures 3 and 4 )

(iii) From 11:00 on October 19, 2016, to $12: 00$ on October 20, 2016, with 1-second increment (see Figures 1 and 2)

The red window in the figures shows the time intervals corresponding to the debates.

3.1. First Debate. An analysis of the retweets' time series using the algorithm (see Section 2.3) made it possible to obtain an estimate of the time moment corresponding to the SOC state, as well as the estimates of the duration of the SubC and the SupC phases. For the entire length of time series $(t \in[0, T])$, the following statistically significant 


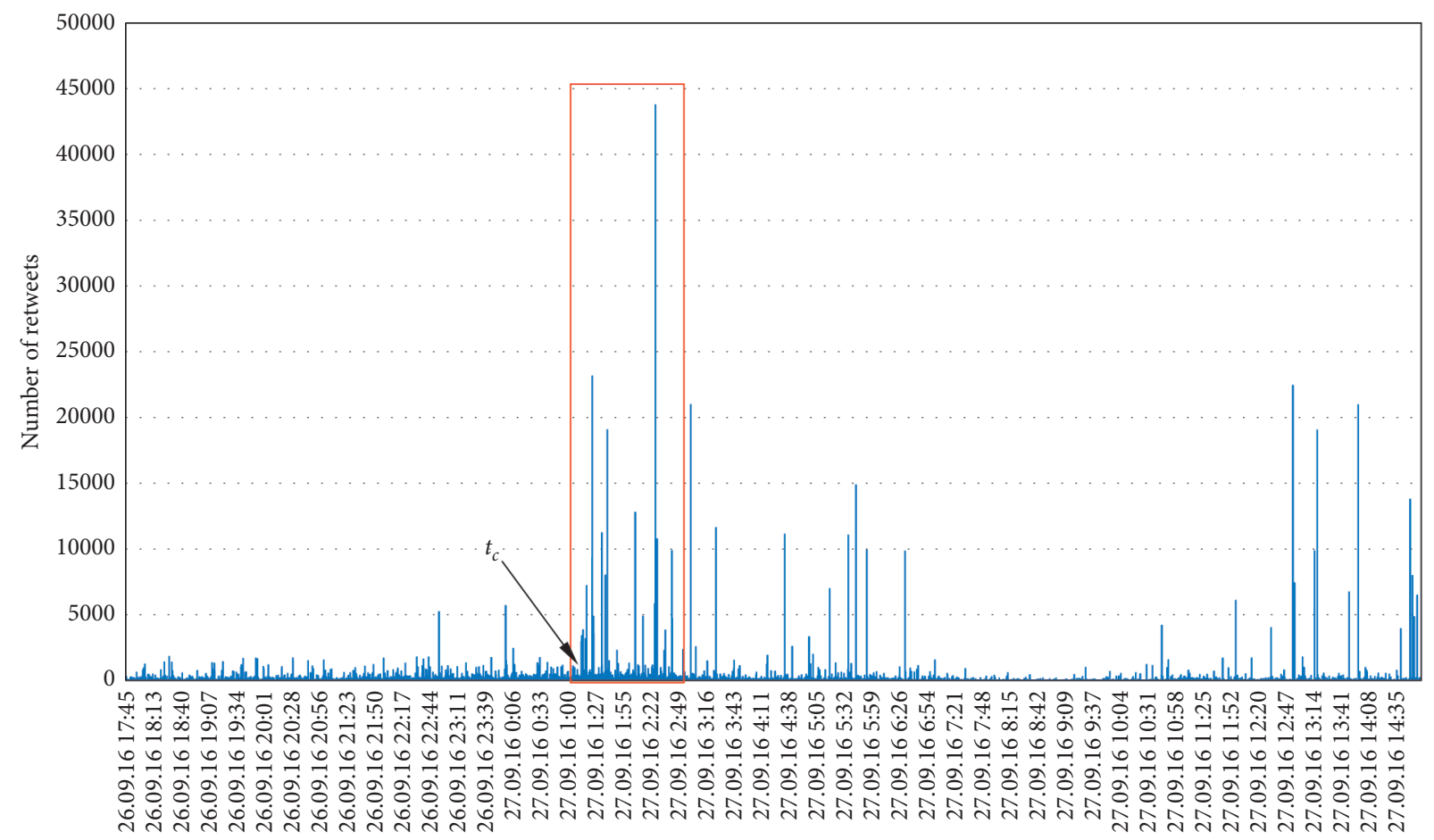

FIGURE 1: The dynamics of retweets corresponding to the first debate. $t_{c}$ denotes the moment of formation of the self-organized criticality state.

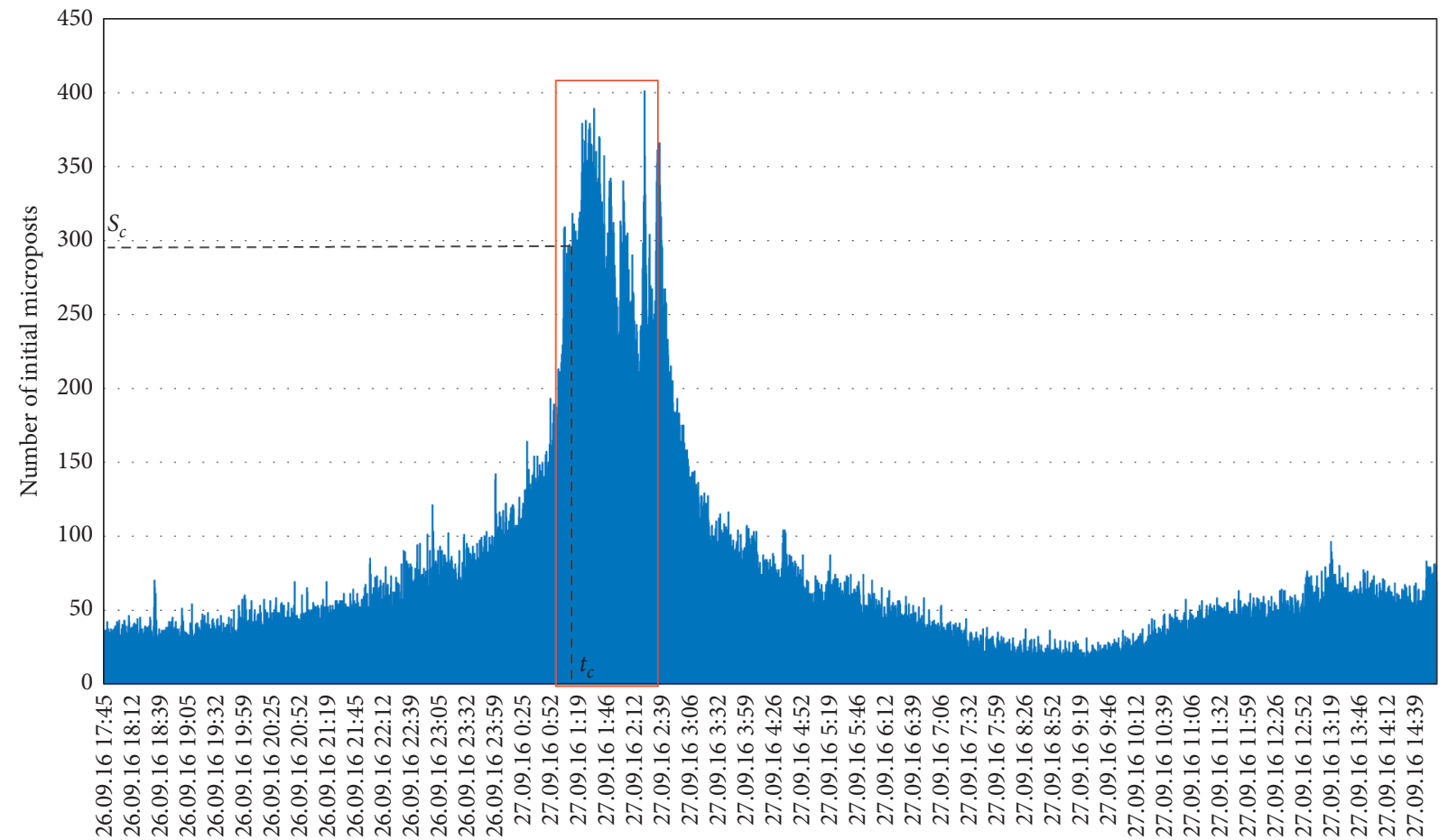

FIGURE 2: The dynamics of retweets corresponding to the first debate. $t_{c}$ denotes the moment of formation of the self-organized criticality state.

estimates of the critical exponents were obtained: $\{2.01(0.0121), 1.23(0.0182), 0.42(0.0201)\} \in\{(1,3),(0.5$, $1.5),(0,1)\}$. The corresponding $p$ values are shown in brackets.
For the timet ${ }^{\prime}$, corresponding to $01: 02$ (September 27), the spectrum $\{3.78(0.7304), 0.09(0.7266), 5.04(0.6345)\} \notin$ $\{(1,3),(0.5,1.5),(0,1)\}$. For the time $t^{\prime \prime}$, corresponding to $01: 24$ (September 27), the spectrum 


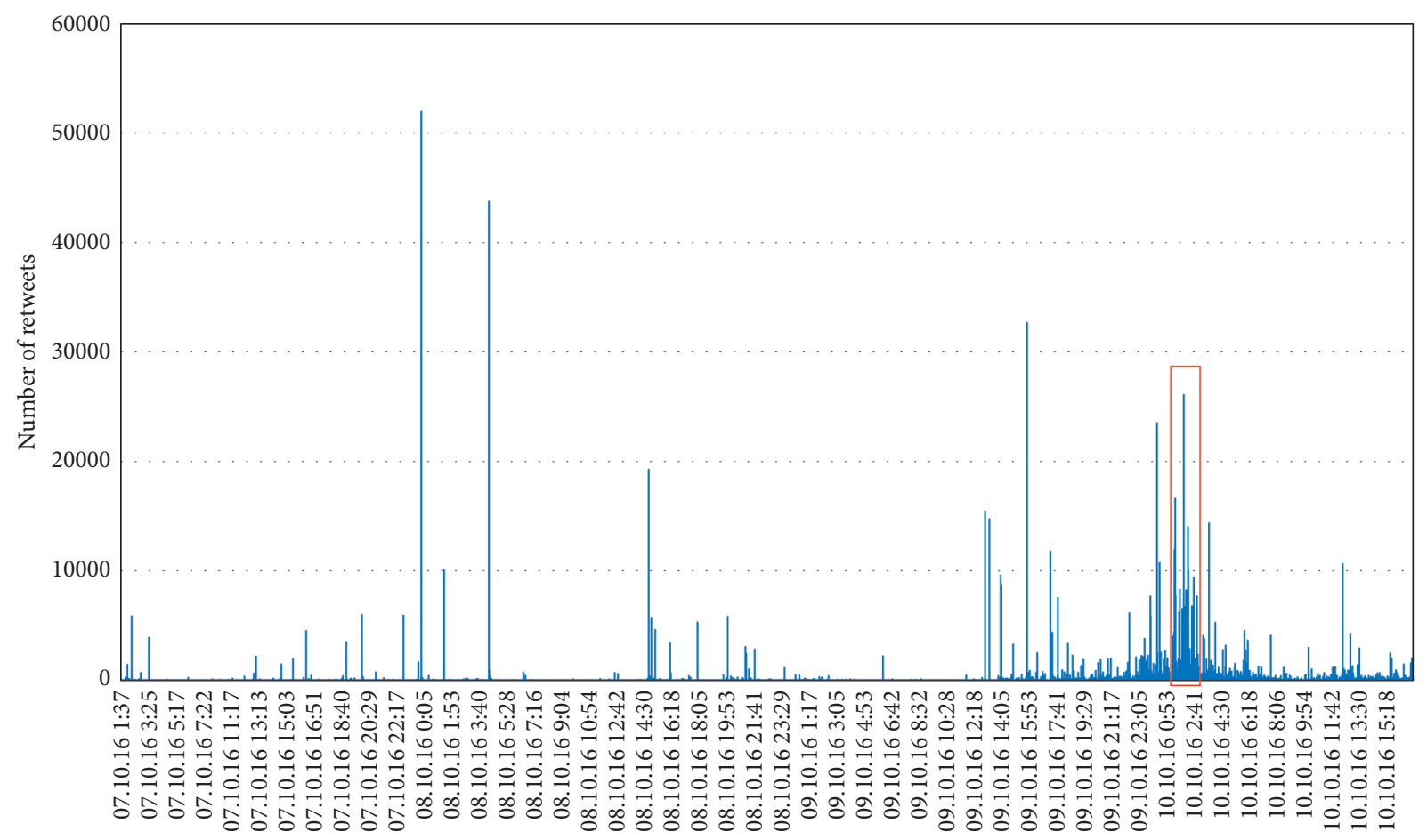

FIgURE 3: The dynamics of retweets corresponding to the second debate.

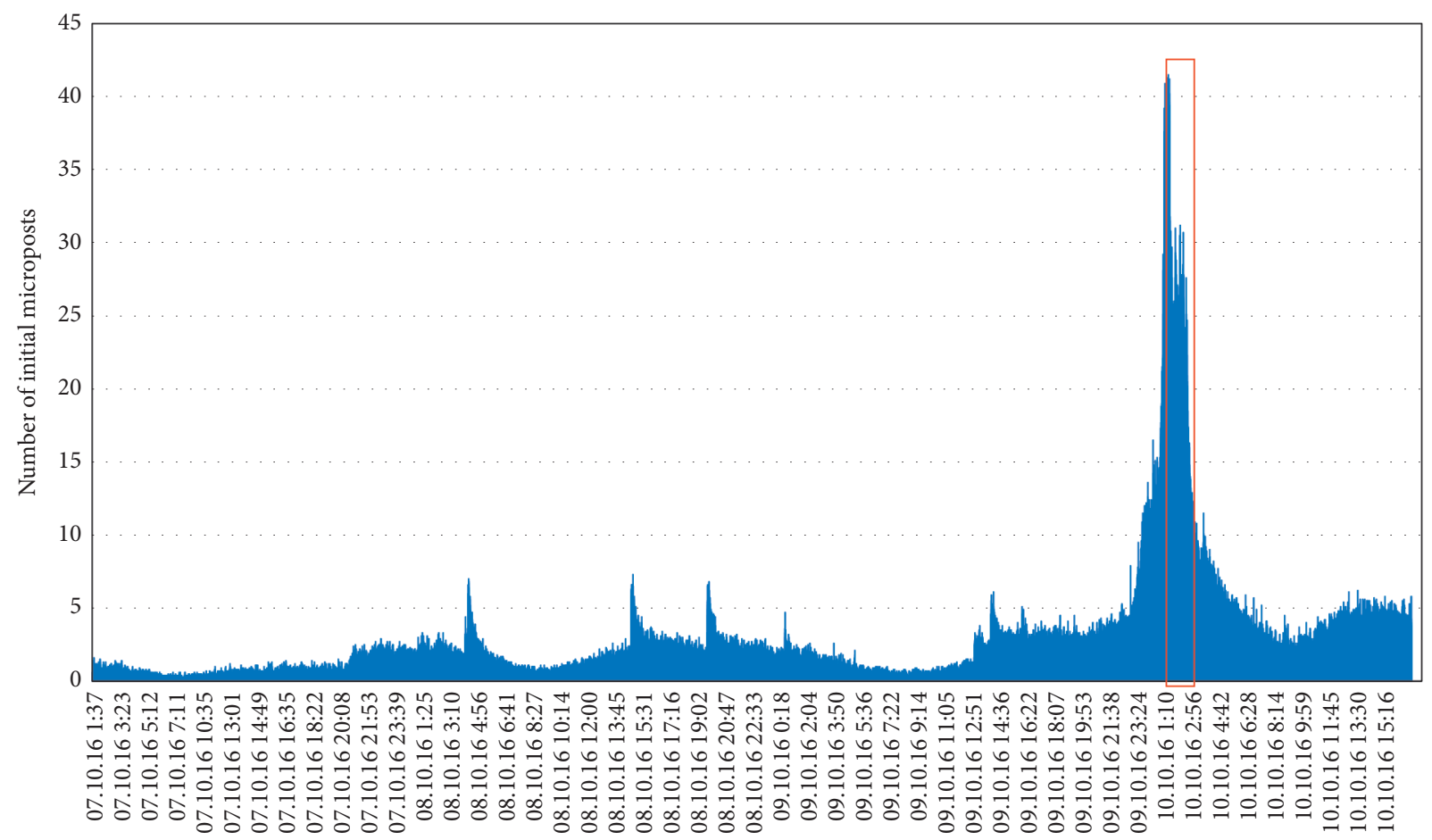

FIgURE 4: The dynamics of initial microposts corresponding to the second debate.

$\{2.01(0.0121), 1.23(0.0182), 0.42(0.0201)\} \in\{(1,3),(0.5$, $1.5),(0,1)\}$. Consequently, SOC state formed at $01: 11$ (September 27). The number of initial microposts is 296 . The duration of the SubC phase is from 17:00 (September 26) to $01: 11$ (September 27). The SupC phase is from $01: 11$ (September 27) to 15:00 (September 27).
Note that the obtained estimates of the duration of critical phases are underestimated because they were obtained from the analysis of available empirical data. To obtain accurate durations, it is necessary to have the time series covering the entire history of the Twitter's evolution related to the information distribution on the network in the 
first debate. However, the estimate $t_{c}$ is not associated with a restriction on the length of the time series.

3.2. Second Debate. The analysis of the retweets' time series corresponding to the second debate failed to establish $t_{c}$. For the entire length of the time series, the following statistically significant estimates of the critical exponents were obtained: $\{2.23(0.0234), 0.98(0.0194), 0.18(0.0209)\} \in\{(1,3),(0.5,1.5$ )$,(0,1)\}$. The scanning of the time series with a fixed boundary both in the direction $T \longrightarrow 0$ (the right boundary $T$ is fixed) and in the opposite direction $0 \longrightarrow T$ (the left boundary 0 is fixed) did not lead to a significant change in the values of the critical exponents. Consequently, the Twitter segment, relevant to the second debate, in the interval from $01: 00$ (October 7) to 17:00 (October 10) is in the SupC phase.

3.3. Third Debate. Similar to the second debate, there is the case with the third debate. For the entire length of the time series, the exponents $\{2.12(0.0312), 1.13(0.0289), 0.34(0.0320)\} \in\{(1,3),(0.5$, $1.5),(0,1)\}$. The values of which do not significantly change when scanning the time row in any direction. Consequently, the Twitter segment, relevant to the third debate, in the interval from 11:00 (October 19) to 15:00 (October 20) is in the SupC phase.

\section{Discussion}

The most important result of our research is the observed selforganization of the electorate in the critical state and, consequently, the ability of such electoral communities to generate information avalanches. Avalanches are bursts of creation, transmission, and reproduction of information in the retweets' form. For the electoral community's self-organization into the SOC state, its members must perceive and respond to each other's microposts. In other words, all community members must be linked by reflexivity.

The existence of the high-level reflexivity is a prerequisite for the functioning of the electoral community in the SOC regime. Indeed, numerous feedback loops are formed only in such communities. In addition, only such communities can be considered as the emergent systems. Integrity and reflexivity determine a high level of community members' involvement.

Thus, only electoral communities, which have been formed as a result of the self-organization of real users, are entering a critical state (see the paper [58]). These communities are not formed by mechanical aggregates of virtual units or by centrally controlled groups. Indeed, it seems unlikely that about five virtual and centrally controlled primary sources could generate more than 5,000 retweets (see Figures 3-6).

Therefore, found values $\{\alpha, \beta, \gamma\} \in\{(1,3),(0.5,1.5)$, $(0,1)\}$ for a certain interval of the retweet time series of the first debate and for the entire length of the retweet time series of the second and third debates are not only the SupC phase identifier but also the identifier of the existence of real electoral communities. This result is very important because it partially refutes numerous claims about the fundamental role of social bots in debate and, moreover, in the results of the 2016 United States Presidential Election (e.g., see the papers [58-60]). We do not deny the existence of the bots and the fact that they distributed the information, but we consider their role as grossly overrated.

4.1. First Debate. The time interval of the social network evolution, in which its self-organization into the critical state is observed, corresponds to the first debate (see Figures 1 and 2). Let us consider one of the possible mechanisms for the transition of the social network in the SOC state from the SubC phase with subsequent evolution into SupC phase. This mechanism seems to us the most reliable, since it is based on the hierarchically subordinate distribution of information in the form of retweets on Twitter. In addition, there is some strong evidence that the dissemination of information in hierarchical structures is avalanche-like (see the papers [61-66]).

A hierarchical organizational structure for Twitter showing the distribution of retweets is shown in Figure 7. Retweet distribution trees, shown with the same colour, are initiated by users of the social network of the same level. Such users are the primary sources (sources of initial microposts) for the distribution of information. The stochastic dynamics of the number of such users is shown in Figures 2, 4, and 6 .

Twitter is an open system. Therefore, the qualitative and quantitative features of the retweets distribution on the network are determined not only by local interactions between network users, but also by external influence on it. First of all, this influence is the inflow of information from the mass media. In the time interval $\left(0, t_{c}\right)$, corresponding to the chaotic SubC phase, information pumping and local interactions between users are not enough for the network to reach the SOC state. This phase is characterized by the existence of many subcommunities that are not linked by retweet interactions (see "SubC phase" in Figure 7). Each of these subcommunities generates small chains of retweets that dissipate over time. Stochastic fluctuations in the number of retweets in the interval $\left(0, t_{c}\right)$ (see Figure 1$)$ correspond to absolutely chaotic white noise $(\beta \cong 0)$, also characterized by the absence of long-term patterns in time $(\gamma \notin(0,1))$ and fluctuations of the relatively small amplitude $(\alpha \notin(1,3))$. Users of individual subcommunities do not perceive and do not respond to each other's microposts, and therefore self-organization into the critical state with the emergence of avalanche-like retweet activity is not possible. Even an increase in the number of primary distribution sources of retweet chains in the interval $\left(0, t_{c}\right)$ (see Figure 2) does not allow the network to self-organize into the critical state.

It should be noted that until 01:11 (September 27) there were no significant resonant events relevant to the first debates. Consequently, self-organization of the network into the critical state, as well as the loss of network stability at time $t_{c}$ as a result of external information pumping, is not also possible. 


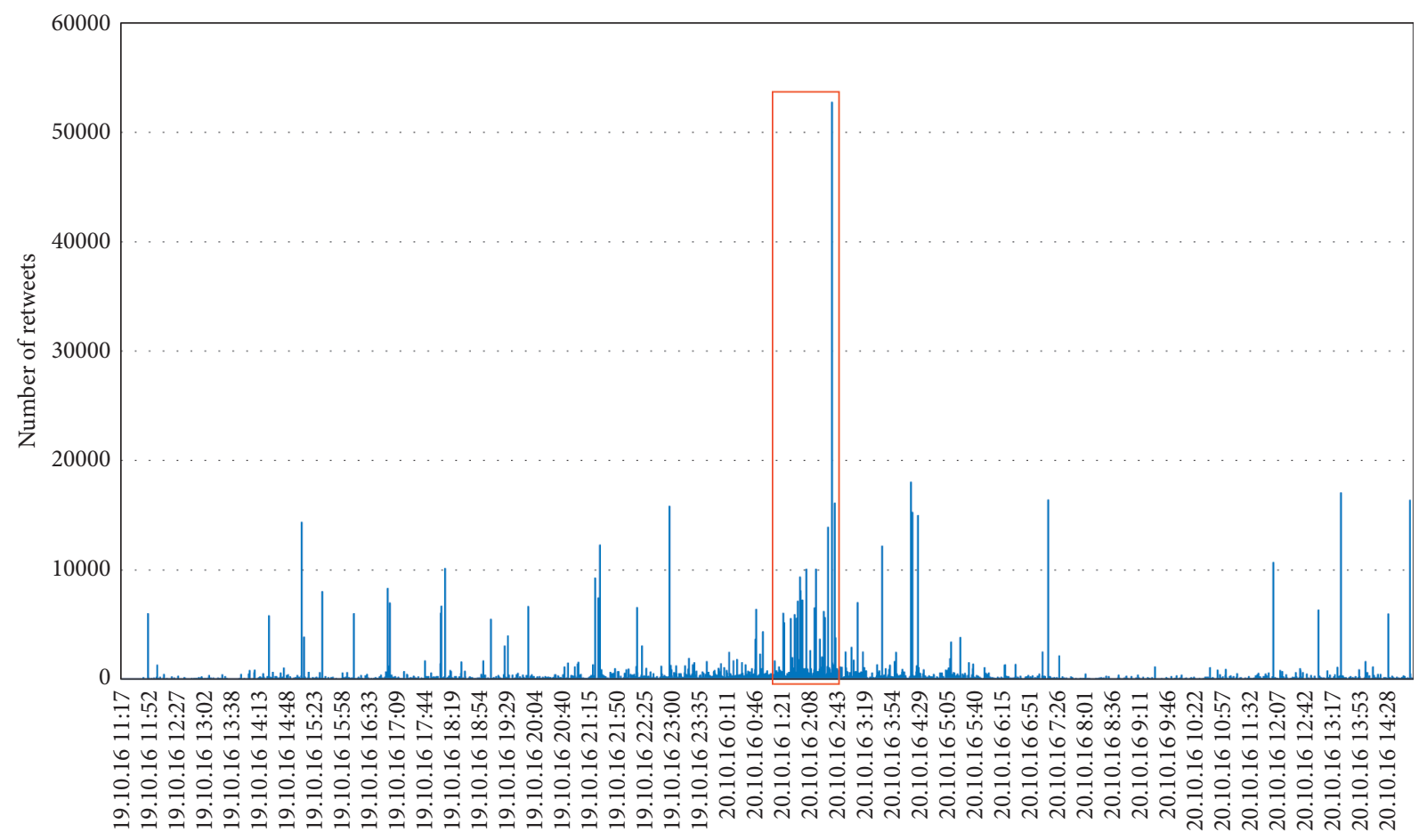

FIGURE 5: The dynamics of retweets corresponding to the third debate.

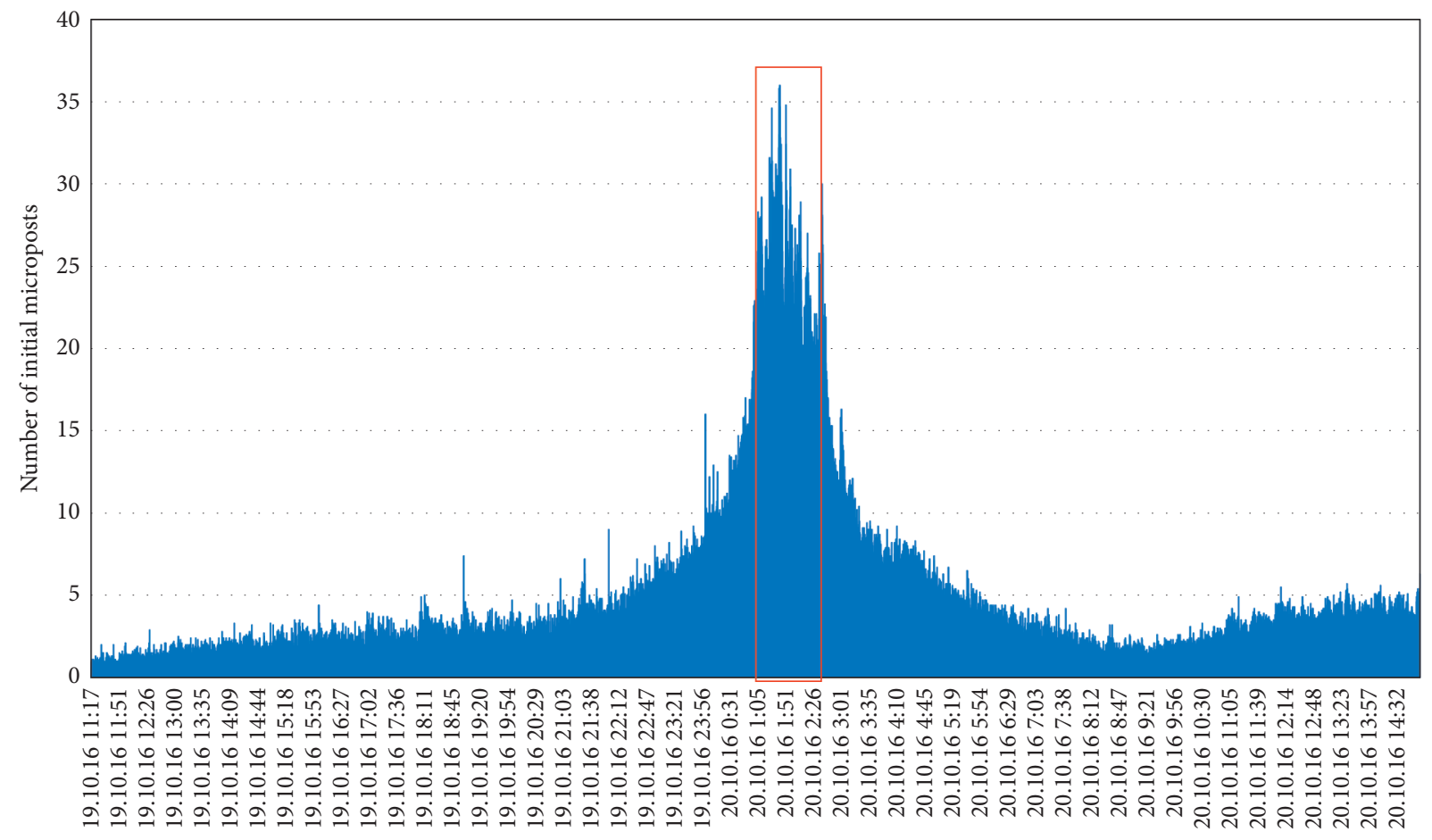

FIgURE 6: The dynamics of initial microposts corresponding to the third debate.

The situation changes fundamentally when the number of initial microposts (primary sources of information distribution) increases to the critical value $S_{c}=296$ (see Figure 2). This number of users is enough to generate avalanche-like retweet streams on the network at time $t_{c}$, corresponding to $01: 11$ (September 27). Twitter state at time $t_{c}$ is the SOC state; this state is unstable to external influences. So, any insignificant external informational influence can bring social networks into the stable SupC phase. Starting from the moment $t_{c}$, the hierarchical organizational structure of its users is formed on the social network, in which avalanches of retweets can spread. Moreover, the 


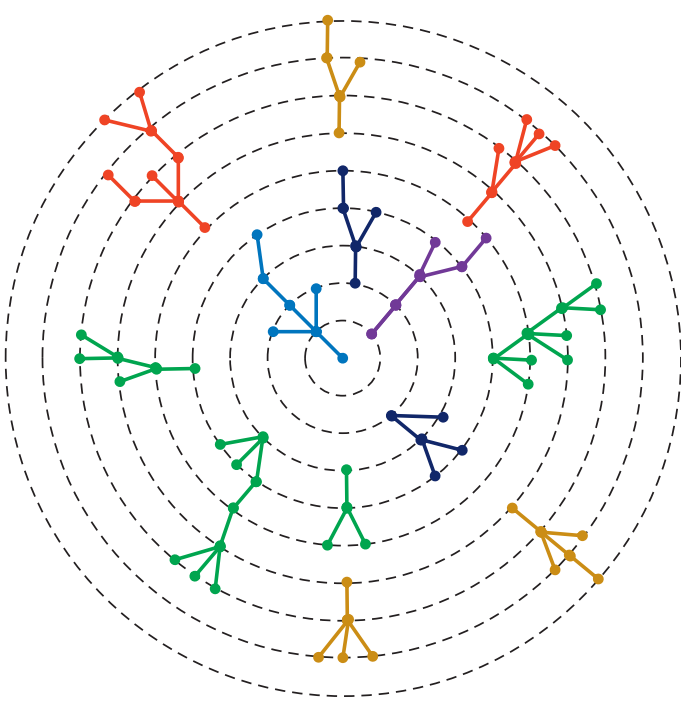

(a)

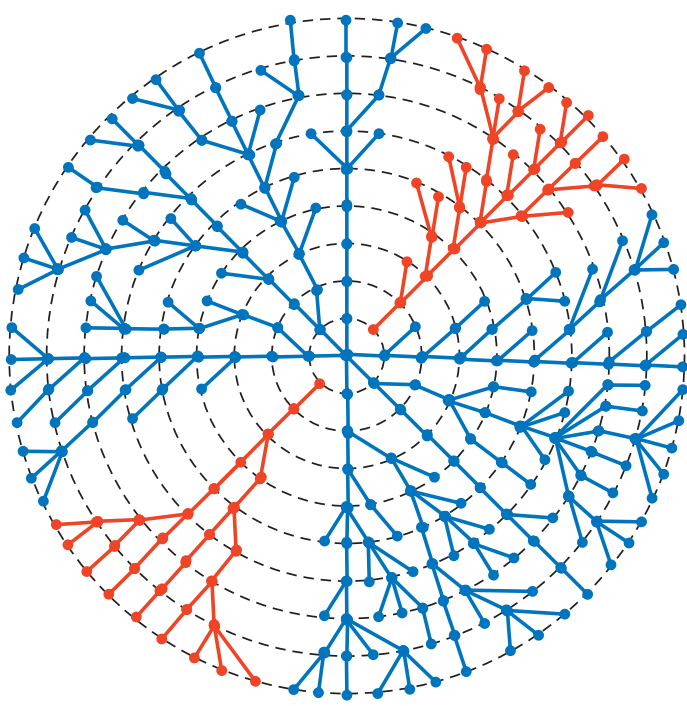

(b)

Figure 7: Hierarchical organizational structure of Twitter retweet distribution, corresponding to chaotic and ordered phases. (a) Subcritical phase. (b) Supercritical phase.

increase in the number of retweets on the network is not only due to the increase in the number of primary distribution sources of the retweet chain. Avalanche-like distribution of retweets is also possible if there is relatively small number of primary sources. Thus, less than 10 primary sources are capable of generating more than 5,000 retweets (see Figures 3-6). The most reasonable explanation for this phenomenon, which is consistent with the way of Twitter functioning, is the existence of highly organized hierarchical user structures (see "SupC phase" in Figure 7). For example, one influencer (primary source) is enough to generate the avalanche of retweets. Such an avalanche is shown in blue in Figure 7 . The primary source is in the center of the circles representing the hierarchical levels.

Of course, we are aware that this is only a reasonable hypothesis, the confirmation of which requires an analysis of the network at the level of detailed interaction of its users.

The SupC phase is resistant to external information influences. Such influences only affect the amplitude of stochastic fluctuations in the number of retweets and do not allow the retweet flows to fade in time and space. Also, in the SupC phase, stochastic fluctuations in the number of primary sources do not affect the existence of avalanche-like retweet activity on the network.

4.2. Second and Third Debates. Analysis of the retweet time series and the initial micropost time series (see Figures 3-6) for the second and third debates, depicting retweet activity on the network, failed to identify the SOC state. The Twitter segment, in which information is distributed, relevant to this debate is in the SupC phase. In our opinion, the reason why we were not able to identify the SOC state is not the limitation of our algorithm. Also, this is not due to the significantly lower number of initial microposts compared to the first debate (see Figures 4 and 6). Note that it makes sense to consider the formation of critical phases and states only in the Twitter segment, corresponding to all three debates. Only separate time series are available to us, but we need to analyze the time series from 17:00 (September 26) to 15:00 (October 20) to obtain a global scheme of the network evolution. In our opinion, we were not able to detect more than one SOC state due to this reason. We do not exclude the fact that during all three debates there is only one SOC state, which formed at 01:11 (September 27).

Twitter evolved into the SupC phase as a result of pumping by information related to various relevant debate events until 15:00 on October 20, 2016. Indeed, starting from this moment in time, many resonant events occurred that affected the dynamics of retweets and prevented the network from going into the relaxation phase. The network was regularly pumped by the information about such events and this made it possible for the network to be in the SupC phase for a long time. Such events include the official accusation of Russia of trying to interfere in the election process, the vulgar comments of one of the candidates, and Podesta e-mail leaks.

\section{Conclusions}

The proposed algorithm allowed to identify the time intervals of critical phases and the time of Twitter self-organization into the critical state. The algorithm is based on the analysis of the retweet activity dynamics, formalized by the retweet time series, and does not require the information about the details of interactions between the social network users.

The results obtained lead to the following conclusions:

(1) Self-organization of Twitter into a critical state occurs during the period of the first debate from the chaotic subcritical phase with an increase in the number of primary sources of retweets to a value of 
approximately 296 . The transition to the critical state could not occur as a result of external significant information pumping of the social network in the absence of informational resonant events while the social network was in the subcritical phase. The reason for the transition to the critical state is the self-organization of the electoral community.

(2) Twitter is in the supercritical phases during the second and third debates. Prior to these phases, subcritical phases and self-organized critical states formed either during the period of the first debate or after the end of the first debate. In the first case, the subcritical phase and the self-organized critical state are the only precursors to the supercritical phases corresponding to the second and third debates. It is necessary to analyze a single retweet time series without interval breaks corresponding to all three debates to justify the second scenario.

(3) The most reliable scenario of Twitter self-organization into the critical state, corresponding to the fundamental principles of the network's functioning, is the formation of the hierarchically organized structure of real users of the network's electoral community as an environment for the spread of the avalanche of retweets.

All studies that are known to us (e.g., see the papers [32-39]), which are devoted to the SOC detection on the social networks, are based on the spectral analysis of the time series in order to detect $1 / f$ noise in them. In such studies, $1 / f$ noise is the only SOC identifier. In addition, except for the study by Zhao with coauthors [67], we are not aware of any studies devoted to the identification of the critical phases and state of the social networks. In these studies, time series analysis is performed for its entire length and for the certain time interval. As shown in Section 4, this analysis is not enough to gain an understanding of the overall nonlinear dynamics of the system, which is characterized by the SOC. Indeed, the existence of $1 / f$ noise in the certain time interval of the time series can be considered only as the existence of the SupC phase in this interval, although it does not guarantee such an existence at all. This is due to the fact that the use of $1 / f$ noise as the only identifier of the SupC phase is reasonable for some model time series, for which the relationship has been established between all three criticality indicators $\{\alpha, \beta, \gamma\}$ (e.g., see the papers [56, 57]). It is possible to limit by the assessment of one of the criticality indicators only for such time series, but for real time series such reliable relationships have not been established. The proposed algorithm is devoid of the indicated drawback. In addition, the algorithm makes it possible to get a complete picture of the time intervals of the critical phases and the moment of the critical state emergence on Twitter.

Despite the indicated capabilities of the algorithm, it has one drawback. The algorithm does not allow to identify the time interval corresponding to the critical deceleration. This phenomenon is caused by a decrease in the frequency of fluctuations in the number of retweets when the number of primary sources (users) of the retweets' distribution chain on the network is near its critical value. Considering that the presence of the critical deceleration time interval precedes the emergence of the SOC state, the appearance of such the interval can be considered as the identifier for early detection of the SOC state.

In conclusion, we briefly consider the possible practical applications of the algorithm. The emergence of the SOC state on Twitter is only possible as a result of the self-organization of the real users' electoral community (see Section 4). Indeed, it seems impossible that around five virtual and centrally controlled primary sources could generate over 5,000 retweets, as shown in Section 4. Thus, as a result of the SOC state detection, we can conclude about the presence or absence of the avalanches of retweets in the SupC phase networks created by social bots. Such a conclusion may provide at least a partial answer to the question of external interference in political elections. Finally, it is known that the network behaves almost unpredictably being in the SOC state. Indeed, Twitter is capable of generating avalanches of retweets of any size being in the SupC phase. This casts doubt on the possibility of the effective forecast of the electoral communities' retweet activity. Twitter's presence in the SOC state can be considered as the basis for decision-making to control the SOC by the election headquarters. Nowadays, several methods have been developed to control the SOC in the systems of different origins (e.g., see the papers [68-70]), the use of which can help the campaign team in decision-making. A detailed consideration of such methods and their application in the election campaign is beyond the scope of our study.

\section{Data Availability}

Previously reported Tweet IDs data used to support this study are available at https://doi.org/10.7910/DVN/PDI7IN. This prior study (and data set) is cited at relevant place within the text as [55].

\section{Conflicts of Interest}

The authors declare that there are no conflicts of interest regarding the publication of this paper.

\section{Acknowledgments}

This work was partially funded by the Russian Foundation for Basic Research (Grant no. 20-07-00651).

\section{References}

[1] M. Newman, "The physics of networks," Physics Today, vol. 61, no. 11, pp. 33-38, 2008.

[2] V. Avetisov, A. Gorsky, S. Maslov et al., "Phase transitions in social networks inspired by the Schelling model," Phys. Rev. E, vol. 98, Article ID 032308, 2018.

[3] P. Fronczak, A. Fronczak, and J. A. Hołyst, "Phase transitions in social networks," The European Physical Journal B, vol. 59, no. 1, pp. 133-139, 2007. 
[4] W. Li, M. Li, S. Zhou et al., "Phase transitions in normalized cut of social networks," Physics Letters A, vol. 383, pp. 3037-3042, 2019.

[5] M. Woloszyn, D. Stauffer, and K. Kulakowski, "Order-disorder phase transition in a cliquey social network," European Physical Journal B, vol. 57, pp. 331-335, 2007.

[6] L. M. Floria, C. Gracia-Lazaro, J. Gomez-Gardenes et al., "Social network reciprocity as a phase transition in evolutionary cooperation," Physical Review E, vol. 79, Article ID 026106, 2009.

[7] D. Tsarev, A. Trofimova, A. Alodjants et al., "Phase transitions, collective emotions and decision-making problem in heterogeneous social systems," Scientific Reports, vol. 9, 2019.

[8] Y. Murase, H. H. Jo, J. Torok et al., "Structural transition in social networks: the role of homophily," Scientific Reports, vol. 9, p. 4310, 2019.

[9] P. H. Barros, I. Cardoso-Pereira, H. Allende-Cid et al., "Leveraging phase transition of topics for event detection in social media," IEEE Access, vol. 8, pp. 70505-70518, 2020.

[10] X. Zhu, J. Song, J. Meng et al., "Information spreading in social network through explosive percolation theory," Lecture Notes in Computer Science, vol. 11280, pp. 487-497, 2018.

[11] A. Campbell, "Word-of-Mouth communication and percolation in social networks," American Economic Review, vol. 103, no. 6, pp. 2466-2498, 2013.

[12] R. Cohen and S. Havlin, "Percolation in complex networks," In Encyclopedia of Complexity and Systems Science, 2009.

[13] Z. Zhang, L. Cui, Z. Pan et al., "A triad percolation method for detecting communities in social networks," Data Science Journal, vol. 17, p. 30, 2018.

[14] F. Morone, B. Min, L. Bo et al., "Collective Influence Algorithm to find influencers via optimal percolation in massively large social media," Scientific Reports, vol. 6, Article ID 30062, 2016.

[15] X. Teng, S. Pei, F. Morone et al., "Collective influence of multiple spreaders evaluated by tracing real information flow in large-scale social networks," Scientific Reports, vol. 6, Article ID 36043, 2016.

[16] B. Barros, A. Fernández-Zubieta, R. Fidalgo-Merino, and F. Triguero, "Scientific knowledge percolation process and social impact: a case study on the biotechnology and microbiology perceptions on Twitter," Science and Public Policy, vol. 45 , no. 6 , pp. 804-814, 2018.

[17] P. Bak, C. Tang, and K. Wiesenfeld, "Self-organized criticality,” Physical Review A, vol. 38, no. 1, pp. 364-374, 1988.

[18] N. Kalinin, A. Guzmán-Sáenz, Y. Prieto, M. Shkolnikov, V. Kalinina, and E. Lupercio, "Self-organized criticality and pattern emergence through the lens of tropical geometry," Proceedings of the National Academy of Sciences, vol. 115, no. 35, pp. E8135-E8142, 2018.

[19] N. W. Watkins, G. Pruessner, S. C. Chapman, N. B. Crosby, and H. J. Jensen, " 25 Years of self-organized criticality: concepts and controversies," Space Science Reviews, vol. 198, no. 1-4, pp. 3-44, 2016.

[20] Y. Wang, H. Fan, W. Lin et al., "Growth, collapse and selforganized criticality in complex networks," Sci Rep, vol. 6, Article ID 24445, 2016.

[21] N. V. Antonov, N. M. Gulitskiy, P. I. Kakin, and G. E. Kochnev, "Effects of turbulent environment on selforganized critical behavior: isotropy vs. Anisotropy," Universe, vol. 6, no. 9, p. 145, 2020.

[22] K. Mahmoodi, B. J. West, and P. Grigolini, "Self-organizing Complex Networks: individual versus global rules," Front Physiol, vol. 8, p. 478, 2017.
[23] B. J. West, G. F. Massari, G. Culbreth et al., "Relating size and functionality in human social networks through complexity," Proceedings of the National Academy of Sciences, vol. 117, pp. 18355-18358, 2020.

[24] D. Zhukov, K. Kunavin, and S. Lyamin, "Online rebellion: self-organized criticality of contemporary protest movements," SAGE Open, vol. 10, pp. 1-22, 2020.

[25] I. Lymperopoulos and G. Lekakos, "Analysis of social network dynamics with models from the theory of complex adaptive systems," In IFIP Advances in Information and Communication Technology, vol. 399, pp. 124-140, 2013.

[26] B. Tadic, V. Dankulov, and R. Melnik, "Mechanisms of selforganized criticality in social processes of knowledge creation," Physical Review E, vol. 96, Article ID 032307, 2017.

[27] I. Shimada and T. Koyama, "A theory for complex systems social change: an application of a general 'criticality' model," Interdisciplinary Description of Complex Systems, vol. 13, no. 3, pp. 342-353, 2015.

[28] D. Antoniades and C. Dovrolis, "Co-evolutionary dynamics in social networks: a case study of Twitter," Computational Social Networks, vol. 2, pp. 1-21, 2015.

[29] A. Casero-Ripollés, "Research on political information and social media: key points and challenges for the future," $\mathrm{El}$ Profesional de la Información," Information Professional, vol. 27, no. 5, pp. 964-974, 2018.

[30] J. Stromer-Galley, Presidential Campaigning in the Internet Age, Oxford University Press, Oxford, UK, 2019.

[31] N. Barabash and D. Zhukov, "Can self-organized criticality theory help identify political mobilization on social media?" Journal for Communication Studies, vol. 13, pp. 155-177, 2020.

[32] Q. K. Meng, "Self-organized criticality in small-world networks based on the social balance dynamics," Chinese Physics Letters, vol. 28, Article ID 118901, 2011.

[33] M. Aguilera, I. Morer, and X. Barandiaran, "Quantifying political self-organization in social media. Fractal patterns in the Spanish 15M movement on twitter," in Proceedings of the 12th European Conference on Artificial Life, pp. 395-402, Sicily, Italy, September 2013.

[34] I. Kirichenko, V. Bulakh, and T. Radivilova, "Fractal time series analysis of social network activities," in Proceedings of the IEEE 4th International Scientific-Practical Conference Problems of Infocommunications, pp. 456-459, Kharkiv, Ukraine, October 2017.

[35] T. De Bie, J. Lijffijt, and C. Mesnage, "Detecting trends in twitter time series," in Proceedings of the IEEE 26th International Workshop on Machine Learning for Signal Processing, pp. 1-6, September 2016, https://doi.org/10.1109/MLSP.2016. 7738815.

[36] D. R. Bild, Y. Liu, R. P. Dick, Z. M. Mao, and D. S. Wallach, "Aggregate characterization of user behavior in Twitter and analysis of the retweet graph," ACM Transactions on Internet Technology, vol. 15, no. 1, pp. 1-24, 2015.

[37] C. Remy, N. Pervin, and F. Toriumi, "Information diffusion on twitter: everyone has its chance, but all chances are not equal," in Proceedings of the IEEE International Conference on Signal-Image Technology and Internet-Based Systems, Kyoto, Japan, December 2013.

[38] J. P. Gleeson and R. Durrett, "Temporal profiles of avalanches on networks," Nature Communications, vol. 8, p. 1227, 2017.

[39] C. Liu, X.-X. Zhan, Z.-K. Zhang et al., "How events determine spreading patterns: information transmission via internal and external influences on social networks," New Journal of Physics, vol. 17, Article ID 113045, 2015. 
[40] P. Zhang, X. Wang, and B. Li, "On predicting Twitter trend: factors and models," in Proceedings of the 2013 IEEE/ACM International Conference on Advances in Social Networks Analysis and Mining, pp. 1427-1429, Niagara Falls, ON, Canada, August 2013.

[41] A. Das, M. Roy, S. Dutta et al., "Predicting trends in the twitter social network: a machine learning approach," In Lecture Notes in Computer Science, vol. 8947, pp. 570-581, 2013.

[42] J. Bollen, H. Mao, and X. Zeng, "Twitter mood predicts the stock market," Journal of Computational Science, vol. 2, no. 1, pp. 1-8, 2011.

[43] L. Montesinos, S. J. P. Rodríguez, M. Orchard, and S. Eyheramendy, "Sentiment analysis and prediction of events in TWITTER," in Proceedings of the 2015 CHILEAN Conference on Electrical, Electronics Engineering, Information and Communication Technologies (CHILECON), pp. 903-910, Santiago, Chile, 2015.

[44] J. M. Soler, F. Cuartero, and M. Roblizo, "Twitter as a tool for predicting elections results," in Proceedings of the 2012 IEEE/ ACM International Conference on Advances in Social Networks Analysis and Mining, pp. 1194-1200, Istanbul Turkey, August 2012.

[45] O. Oyebode and R. Orji, "Social media and sentiment analysis: the nigeria presidential election 2019," in Proceedings of the 2019 IEEE 10th Annual Information Technology, Electronics and Mobile Communication Conference, pp. 0140-0146, Vancouver, Canada, October 2019.

[46] J. Ramteke, S. Shah, D. Godhia, and A. Shaikh, "Election result prediction using twitter sentiment analysis," in Proceedings of the 2016 International Conference on Inventive Computation Technologies, pp. 1-5, Coimbatore, India, August 2016.

[47] B. Bansal and S. Srivastava, "On predicting elections with hybrid topic based sentiment analysis of tweets," Procedia Computer Science, vol. 135, pp. 346-353, 2018.

[48] S. M. Srinivasan, R. S. Sangwan, C. J. Neill, and T. Zu, "Twitter data for predicting election results: insights from emotion classification," IEEE Technology and Society Magazine, vol. 38, no. 1, pp. 58-63, 2019.

[49] X. Wang, L. Tokarchuk, and S. Poslad, "Identifying relevant event content for real-time event detection,", in Proceedings of the 2014 IEEE/ACM International Conference on Advances in Social Networks Analysis and Mining, pp. 395-398, Beijing, China, August 2014.

[50] S. Yousefinaghani, R. Dara, Z. Poljak et al., "“The assessment of Twitter's potential for outbreak detection: avian influenza case study," Scientific Reports, vol. 9, Article ID 18147, 2019.

[51] J. P. Singh, Y. K. Dwivedi, N. P. Rana, A. Kumar, and K. K. Kapoor, "Event classification and location prediction from tweets during disasters," Annals of Operations Research, vol. 283, no. 1-2, pp. 737-757, 2019.

[52] S. N. Dorogovtsev, A. V. Goltsev, and J. F. F. Mendes, "Critical phenomena in complex networks," Reviews of Modern Physics, vol. 80, no. 4, pp. 1275-1335, 2008.

[53] A. Dmitriev, V. Dmitriev, and S. Balybin, "Self-organized criticality on twitter: phenomenological theory and empirical investigation based on data analysis results," Complexity, vol. 2019, Article ID 8750643, 16 pages, 2019.

[54] R. Nishi, T. Takaguchi, and K. Oka, "Reply trees in Twitter: data analysis and branching process models," Social Networks Analysis and Mining, vol. 6, pp. 1-13, 2016.

[55] J. Littman, L. Wrubel, and D. Kerchner, 2016 United States Presidential Election Tweet Ids, Harvard Dataverse, V3, 2016, https://dataverse.harvard.edu/dataset.xhtml? persistentId= doi:10.7910/DVN/PDI7IN.
[56] S. B. Lowen and M. C. Teich, "Fractal renewal processes generate 1/fnoise," Physical Review E, vol. 47, no. 2, pp. 992-1001, 1993.

[57] S. Maslov, M. Paczuski, and P. Bak, "Avalanches and1fNoise in evolution and growth models," Physical Review Letters, vol. 73, no. 16, pp. 2162-2165, 1994.

[58] A. Bovet and H. Makse, "Influence of fake news in Twitter during the 2016 US presidential election," Nature Communications, vol. 10, pp. 1-14, 2019.

[59] C. Shao, G. Ciampaglia, O. Varol et al., "The spread of lowcredibility content by social bots," Nature Communications, vol. 9, pp. 1-9, 2018.

[60] A. Bessi and E. Ferrara, "Social bots distort the 2016 U.S. Presidential election online discussion," First Monday, vol. 21, pp. 1-14, 2016.

[61] P. Moriano, J. Finke, and Y. Ahn, "Community-based event detection in temporal networks," Scientific Reports, vol. 9, p. 4358, 2019.

[62] J. P. Gleeson, J. A. Ward, K. P. O'Sullivan et al., "Competitioninduced criticality in a model of meme popularity," Physical Review Letters, vol. 112, Article ID 048701, 2014.

[63] J. P. Gleeson, K. P. O’Sullivan, R. A. Banos et al., "Effects of network structure, competition and memory time on social spreading phenomena," Physical Review X, vol. 6, Article ID 021019, 2016.

[64] S. Morse, M. C. Gonzalez, and N. Markuzon, "Role of persistent cascades in diffusion," Physical Review E, vol. 99, pp. 12323-12331, 2019.

[65] J. Liu, Z. He, and Y. Huang, "Hashtag2Vec: learning hashtag representation with relational hierarchical embedding model," in Proceedings of the Twenty-Seventh International Joint Conference on Artificial Intelligence, pp. 3456-3462, Stockholm, Sweden, July 2018.

[66] M. J. Krawczyk, P. Oświęcimka, and K. Drożdż, “Ordered avalanches on the bethe lattice," Entropy, vol. 21, no. 10, p. 968, 2019.

[67] L. Zhao, W. Li, C. Yang et al., "Multifractality and network analysis of phase transition," PLoS ONE, vol. 12, Article ID e0170467, 2017.

[68] D. O. Cajueiro and R. F. S. Andrade, "Controlling self-organized criticality in sandpile models," Phys. Rev. E, vol. 81, no. R, Article ID 015102, 2010.

[69] D. O. Cajueiro and R. F. S. Andrade, "Controlling self-organized criticality in complex networks," The European Physical Journal B, vol. 77, no. 2, pp. 291-296, 2010.

[70] H. Hoffmann and D. W. Payton, "Suppressing cascades in a self-organized-critical model with non-contiguous spread of failures," Chaos, Solitons and Fractals, vol. 67, pp. 87-93, 2014. 